\section{Pacific Northwest}

National Laboratory

Operated by Battelle for the

U.S. Department of Energy

\author{
Anthony Peurrung \\ Richard Arthur \\ Bruce Geelhood \\ Randy Scheele \\ Robert Elovich \\ Sharon Pratt
}

March 7, 2000

Prepared for the U.S. Department of Energy under Contract DE-AC06-76RLO 1830 


\section{DISCLAIMER}

This report was prepared as an account of work sponsored by an agency of the United States Government. Neither the United States Government nor any agency thereof, nor Battelle Memorial Institute, nor any of their employees, makes any warranty, express or implied, or assumes any legal liability or responsibility for the accuracy, completeness, or usefulness of any information, apparatus, product, or process disclosed, or represents that its use would not infringe privately owned rights. Reference herein to any specific commercial product, process, or service by trade name, trademark, manufacturer, or otherwise does not necessarily constitute or imply its endorsement, recommendation, or favoring by the United States Government or any agency thereof, or Battelle Memorial Institute. The views and opinions of authors expressed herein do not necessarily state or reflect those of the United States Govermment or any agency thereof.

\section{PACIFIC NORTHWEST NATIONAL LABORATORY \\ operated by \\ BATTELLE \\ for the \\ UNITED STATES DEPARTMENT OF ENERGY \\ under Contract DE-AC06-76RLO 1830}

Printed in the United States of America

Available to DOE and DOE contractors from the

Office of Scientific and Technical Information,

P.O. Box 62, Oak Ridge, TN 37831-0062;

ph: (865) 576-8401

fax: $(865)$ 576-5728

email: reports@adonis.osti.gov

Available to the public from the National Technical Information Service, U.S. Department of Commerce, 5285 Port Royal Rd., Springfield, VA 22161 ph: (800) 553-6847 fax: (703) 605-6900

email: orders@ntis.fedworld.gov

online ordering: http://www.ntis.gov/ordering.htm

This document was printed on recycled paper. 


\section{DISCLAIMER}

Portions of this document may be illegible in electronic image products. Images are produced from the best available original document. 


\title{
Origin of The 871-keV Gamma Ray and the "Oxide" Attribute
}

\author{
Anthony Peurrung, Richard Arthur, Bruce Geelhood, Randy Scheele, \\ Robert Elovich, Sharon Pratt \\ Pacific Northwest National Laboratory \\ $3 / 7 / 00$
}

\begin{abstract}
Summary
This brief paper concludes our study of the origin of the 871-keV observed for many plutonium oxide samples. Analysis of a variety of experimental results confidently establish the following conclusions:

- The $871-\mathrm{keV}$ gamma ray arises solely from the reaction ${ }^{14} \mathrm{~N}(\alpha, p){ }^{17} \mathrm{O}$.

- Even the experimentally inferred maximum possible contribution from the reaction ${ }^{17} O\left(\alpha, \alpha^{\prime}\right)^{17} O^{*}$ would not be measurable in any oxide sample.

- The presence of air within or surrounding the oxide powder leads to a measurable 871-keV gamma-ray flux.

- A nitrogen impurity of roughly $200 \mathrm{ppm}$ would lead to a measurable $871-\mathrm{keV}$ gamma-ray flux. (Samples measured at PNNL did not contain this level of nitrogen impurity.)
\end{abstract}

The implications of these results for arms control are complex. Clearly, the presence of an $871-\mathrm{keV}$ gamma-ray flux indicates that a sample is not metallic. Unfortunately, the converse assertion is far from true. That is, the lack of a measurable 871-keV gammaray flux does not indicate that the sample is metallic. Plutonium in the oxide form can easily be "disguised" via compaction, purification, or atmospheric control. We would suggest that a more appropriate rame for this attribute would be "presence of nitrogen." This new name accurately conveys the strengths and weaknesses of this attribute as we now understand them.

These conclusions were established via a series of spectral measurements of plutonium and americium in a variety of physical states. Acidified solutions, solid oxide powder, and metallic forms were analyzed. The atmosphere surrounding the powder was controlled and varied between argon, air, and nitrogen. Chemical processing was employed on the oxide powder to remove all solid nitrogen impurity. The aqueous solutions were in both hydrochloric and nitric acids of varying molarity.

A comprehensive theoretical analysis of the experimental results is able to consistently explain the full suite of results. While the underlying nuclear reaction cross sections for production of the 871-keV gamma ray in nitrogen and oxygen are not known, the constraints placed upon the necessary cross sections are not unreasonable. 


\section{Introduction}

This work concludes the investigation of the "oxide" attribute of current interest for the characterization of stored plutonium. Originally it was believed that the presence of oxide could be ascertained by measurement of the $871-\mathrm{keV}$ line in a high-resolution gamma-ray spectrum (Hsu, 1982). However, recent work has suggested that the $871-\mathrm{keV}$ gamma ray in plutonium oxide arises from the reaction ${ }^{14} \mathrm{~N}(\alpha, \mathrm{p}){ }^{17} \mathrm{O}$ rather than the inelastic scattering reaction ${ }^{17} \mathrm{O}\left(\alpha, \alpha^{\prime}\right)^{17} \mathrm{O}^{*}$ (Geelhood, 1999; Marlow, 1999;

Peurrung, 1999). This conclusion, though initially surprising, was obtained during efforts to determine the relative importance of americium and plutonium alpha-particle decay for the production of the $871-\mathrm{keV}$ gamma ray. Several questions were raised by previous experiments:

- What role, if any, does ${ }^{17} \mathrm{O}$ have in the generation of the $871-\mathrm{keV}$ gamma ray?

- How does sufficient nitrogen come to be present in plutonium oxide?

- Under what conditions is the $871-\mathrm{keV}$ gamma ray measurable in plutonium oxide?

This paper describes the answers to these questions. We can state confidently that ${ }^{17} \mathrm{O}$ has no significant role in the generation of the $871-\mathrm{keV}$ line for any oxide samples. There are arguably three different ways that nitrogen comes to be present in plutonium oxide. First, nitrogen may be present as an impurity at roughly the 1000 -ppm level or higher. Such nitrogen would lead to an $871-k e V$ gamma-ray flux that is easily measurable under ordinary conditions. Second, immersion of a powder in air causes significant amounts of nitrogen to permeate the pore spaces between the individual oxide particles. Third, a measurable $871-\mathrm{keV}$ gamma-ray flux could arise merely from the interaction of alpha particles (from plutonium decay) with air surrounding a small oxide sample. Thus, a measurable 871-keV gamma-ray flux should be observable only when the oxide either contains significant nitrogen as an impurity, or when air is present within or around an oxide powder. We believe that the $871-\mathrm{keV}$ line will not be observed from oxide under any of the following conditions:

- The oxide is surrounded by a gas such as argon or helium that contains little nitrogen.

- The oxide is compacted and sealed with little extra space for air.

- The oxide is immersed in or dissolved in a liquid such as oil or water that does not contain nitrogen.

The $871-\mathrm{keV}$ peak is not observed from a metal sample because the surface area potentially in contact with surrounding ${ }^{14} \mathrm{~N}$ is much smaller than for an oxide sample. Measures taken to prevent oxidation of metal components (e.g. plating or inert gas atmosphere) also prevent the alpha interaction with ${ }^{14} \mathrm{~N}$.

\section{Experiments}

The work incorporates both the earlier experiments with ${ }^{241} \mathrm{Am}$ and ${ }^{239} \mathrm{Pu}$ dissolved in aqueous solutions of hydrochloric and nitric acids, and newer experiments with plutonium oxide powder. In all cases, a high-resolution gamma-ray spectrometer was used in conjunction with sufficient lead attenuator to reduce dead time to acceptable levels. Counting times ranged from minutes to hours, depending upon the length of time necessary to acquire a spectrum with adequate statistics for measuring and comparing the $871-\mathrm{keV}$ flux. In at least one case a sophisticated low-background cave was used to 
lower background and permit measurement of the $871-\mathrm{keV}$ gamma-ray flux from a rather weak source $\left(11 \mu \mathrm{Ci}\right.$ of $\left.{ }^{239} \mathrm{Pu}\right)$.

The goal of the most recent experiments was to fully characterize the nitrogen within a plutonium oxide sample and quantify its role in the generation of the 871-keV gamma ray. To this end, a sample of roughly 5.2 grams of dry plutonium (weapons-grade with $0.42 \%{ }^{241} \mathrm{Am}$ grown in) oxide power was subjected to a variety of physical and chemical manipulations. The atmosphere surrounding this powder was controlled and variously selected to be ordinary air, pure argon, and pure nitrogen. In order to remove any nitrogen impurities, the powder sample was then subjected to the following sequential steps:

- An oxalate wash followed by drying and calcining ${ }^{1}$ at $1000^{\circ} \mathrm{C}$ to convert any remnant of oxalate or carbonate into oxide. This step also removed some fluoride impurities and should have removed any soluble nitrogen impurities.

- Vigorous Dissolution in sodium hydroxide and hydrogen peroxide ("sodium fusion") followed by acidification in hydrochloric acid.

- Precipitation with oxalate and calcining at $1000^{\circ} \mathrm{C}$.

It should be noted that the physical character of the powder (density, granularity, etc.) was observed to change as a result of these manipulations.

\section{Results}

Although the region of the plutonium spectrum near $871-\mathrm{keV}$ contains many peaks, the $871-\mathrm{keV}$ line can be sufficiently strong that its presence can be determined with high statistical confidence in less than an hour using a typical HPGe spectrometer. Figure 1 shows six spectra taken during the course of this work. Five of the spectra are of the plutonium oxide powder sample under various conditions. The two "pre-wash" spectra are the oxide (as originally received) under nitrogen and argon atmospheres. The "washed" spectrum is under air. Finally, the two "purified" oxide spectra are the final purified powder under nitrogen and argon atmospheres. For comparison, a plutonium bulk-metal spectrum is shown. The metallic plutonium is material with a different mass, isotopic composition, age, and counting geometry. Nevertheless, it serves as a useful comparison spectrum. All spectra, except the Pu metal, have been normalized to the strong 769-keV peak from ${ }^{239} \mathrm{Pu}$, which is not shown in Figure 1. Figure 2 shows essentially the same data as Figure 1, but with the strongest nearby peaks from ${ }^{239} \mathrm{Pu}$ and ${ }^{241} \mathrm{Am}$ shown to illustrate this normalization process. Note the recoil-broadened ${ }^{72} \mathrm{Ge}$ neutron peak that increases in energy starting at $834 \mathrm{keV}$. The feature is due to fast neutrons from the sample that undergo inelastic scatter on ${ }^{72} \mathrm{Ge}$ within the detector crystal itself.

There are a number of peaks in the general region other than the 871-keV peak, as seen in Figure 1. These peaks arise not only from plutonium and americium, but also from

\footnotetext{
${ }^{1}$ N.B. - "Calcine" is the chemical term for heating a refractory material to a high temperature to drive off volatile components or to convert a compound to a simpler chemical form. For the plutonium sample used in this study, the purpose was to convert any nitrate, oxalate, or carbonate to the oxide (Cleveland, 1967). The process here does not involve any reactions with calcium, but rather, the term "calcination" derives historically from the production of lime (calcium oxide) by roasting calcite (i.e, limestone, chalk, or marble as principal sources of calcium carbonate), which decomposes the carbonate radical and drives off the resulting carbon dioxide.
} 
${ }^{228}$ Th daughters, inelastic neutron scattering gamma rays (Zhou, 2000), and even other $(\alpha, n)$ reactions. A listing of the identified peaks is provided in Table 1.

Table 1. Additional Peaks and Associated Branching Fraction as Identified in the 870-keV Region of Figure 1.

\begin{tabular}{|l|c|l|}
\hline Peak & Branching Fraction & Comments \\
\hline $851.72 \mathrm{keV}$ & $2.03 \cdot 10^{-6}$ & Pu-238 \\
$860.30 \mathrm{keV}$ & - & TI-208 (daughter of Th-228) \\
$862.6 \mathrm{keV}$ & $5.3 \cdot 10^{-7}$ & Am-241 \\
$870.8 \mathrm{keV}$ & - & ${ }^{14} \mathrm{~N}(\alpha, \mathrm{p})^{17} \mathrm{O} \quad \leftarrow$ 871-keV Peak of interest \\
$872.7 \mathrm{keV}$ & $7 \cdot 10^{-7}$ & Am-241 \\
$873.94 \mathrm{keV}$ & $5.8 \cdot 10^{-7}$ & $\mathrm{Pu}-240$ \\
$881.0 \mathrm{keV}$ & - & ${ }^{206} \mathrm{~Pb}\left(\mathrm{n}, \mathrm{n}^{\prime}\right)^{206} \mathrm{~Pb}^{\star}$ \\
$883.24 \mathrm{keV}$ & $1.11 \cdot 10^{-6}$ & $\mathrm{Pu}-238$ \\
$890.9 \mathrm{keV}$ & - & ${ }^{19} \mathrm{~F}(\alpha, \mathrm{n})^{22} \mathrm{Na}$ \\
\hline
\end{tabular}

Note that both the 873-keV and 874-keV peaks from Am-241 and Pu-240 are close enough to the $871-\mathrm{keV}$ peak that resolution of the two separately is difficult or impossible. This fact complicates statistical identification of the $871-\mathrm{keV}$ peak when it is weak.

We conclude that the $871-\mathrm{keV}$ flux arises from nitrogen impurities and from nitrogen gas surrounding the powder, as seen in Figures 1 and 2. Clearly the ratio of the 871-keV flux to the 769-keV flux would not change (as was observed) were oxygen the dominant source of $871-\mathrm{keV}$ gamma-ray emission. Instead, we find that the amplitude of the 871$\mathrm{keV}$ peak varies with atmosphere at each of the three stages in our purification process. The effect of our efforts to remove nitrogen impurities in the oxide powder is harder to determine conclusively. There appears to be a decrease in the 871-keV line intensity between the nitrogen-atmosphere spectra before and after the washing and calcining steps. However, the $871-\mathrm{keV}$ line returns again to nearly its original intensity after the dissolution, acidification, and precipitation steps. We believe these seemingly paradoxical results are the result of the fact that the $871-\mathrm{keV}$ line arises primarily from alpha-particle interactions with the nitrogen gas. The powder's density (void fraction) changes after each step as does the amount of powder that adheres to the sides of the container. We thus take this behavior as evidence that gas-phase interactions are of primary importance.

The clearest evidence for the prevalence of gas-phase interactions is shown in Figure 3. This figure shows only the spectra of the purified oxide powder with argon and nitrogen atmospheres. Note that the full spectra are virtually identical except for the $871-\mathrm{keV}$ line. The remaining nitrogen impurity concentration for these spectra should be both greatly reduced relative to the original concentration and completely insignificant in the context of $871-\mathrm{keV}$ gamma-ray generation. Based upon these results it is hard to imagine attributing the $871-\mathrm{keV}$ flux to any source other than alpha-particle interactions with gasphase nitrogen. 
These results are not surprising upon careful consideration. Previous results found that $1.1 \mathrm{mCi}$ of ${ }^{239} \mathrm{Pu}$ in an 8 molar solution of nitric acid emitted the $871-\mathrm{keV}$ gamma ray at a rate of roughly 2.1 per second. The same amount of plutonium emits the $769-\mathrm{keV}$ gamma ray at a rate of 4.6 per second as a result of plutonium decay. We know conclusively that the $871-\mathrm{keV}$ flux from the nitric acid solution arises from nitrogen interactions for two reasons:

1) The flux scaled linearly with the molarity of the nitric acid solution for two different samples.

2) No measurable 871-keV flux was present when americium was dissolved in a hydrochloric acid solution, but is manifest when nitric acid is added.

It is reasonable to expect that the ratio of the $871-\mathrm{keV}$ flux to the $769-\mathrm{keV}$ flux should scale linearly with both the nitrogen concentration and the range of the alpha particle. The alpha-particle range for the aqueous solution is roughly 40 microns. The range in plutonium oxide depends, of course, on the powder density. Although the nominal density of plutonium oxide is roughly $11.5 \mathrm{~g} / \mathrm{cm}^{3}$, the density for our powder was in the range of $3-4 \mathrm{~g} / \mathrm{cm}^{3}$. Consequently, the alpha-particle range in our purified oxide powder was also close to 40 microns. These calculations are based upon Figure 2-8 ("Rangeenergy curves calculated for alpha particles in different materials") of Knoll's text on radiation detection (Knoll, 1989). We hypothesize, therefore, that the ratio of the $871-\mathrm{keV}$ flux to the 769-keV flux for both aqueous acid solutions and fine, low-density oxide powders should scale approximately with the nitrogen concentration. (We neglect differential attenuation for these small samples.) The nitrogen concentration in the $8 \mathrm{M}$ nitric acid solution is $4.8 \times 10^{21} / \mathrm{cm}^{3}$. The presence of air within the powder results in a nitrogen concentration of roughly $3.5 \times 10^{19} / \mathrm{cm}^{3}$. One would therefore expect the line ratio to be roughly 140 times lower in the oxide than in the acid solution. In fact, the ratio was observed to be roughly 18 times lower, leaving a discrepancy of a factor of roughly 7.6.

This additional factor of 7.6 is believed to arise from alpha-particle interactions in the gaseous nitrogen external, rather than internal, to the powder. There is only about $0.8 \mathrm{~cm}^{3}$ of nitrogen gas within the powder, whereas there is roughly $10 \mathrm{~cm}^{3}$ of gas in the space around the powder. Since the range of alpha particles in air is roughly $3.5 \mathrm{~cm}$, the nitrogen reaction will be possible during roughly the first centimeter of a 5-MeV alpha particle's track through the air. While exact calculations are impossible given the unknown distribution of oxide on the sides of the container, it seems clear that such interactions easily account for the bulk of the $871-\mathrm{keV}$ gamma-ray generation.

Two quantitative results of importance can be gathered from these experiments. First, we can state that the nitrogen impurity concentration necessary to lead to $871-\mathrm{keV}$ peak generation of significance is roughly $3 \times 10^{19} / \mathrm{cm}^{3}$, which corresponds to $200 \mathrm{ppm}$. Amounts significantly less than this would not be able to generate a measurable $871-\mathrm{keV}$ flux. Second, we can estimate the maximum contribution to the $871-\mathrm{keV}$ flux that oxygen could account for. Measurement of the hydrochloric acid solution of $86 \mu \mathrm{Ci}$ of americium placed an upper bound of 0.004 photons/second on the $871-\mathrm{keV}$ emission rate. Assuming conservatively that plutonium alpha particles produce $871-\mathrm{keV}$ gamma rays at the same rate as plutonium gamma rays, we can place an upper bound on the oxygen generation rate in our powder sample. Calculations indicate that the $871-\mathrm{keV}$ flux from oxygen can be no more than $10 \%$ of the value observed for the pure oxide powder in a nitrogen atmosphere. Even at this maximum possible level, the flux would be hard to measure in a reasonable time, especially in light of the nearby ${ }^{241} \mathrm{Am}$ and 
${ }^{240} \mathrm{Pu}$ peaks. (These peaks will be more pronounced for plutonium containing more

${ }^{240} \mathrm{Pu}$ than the $6 \%$ for this oxide sample.)

\section{Conclusion}

The experimental data resulting from high resolution spectra of plutonium oxide powder and aqueous solutions of plutonium and americium allow us to conclude that the $871-\mathrm{keV}$ gamma ray observed for many oxide samples always arises from the reaction ${ }^{14} \mathrm{~N}(\alpha, \mathrm{p}){ }^{17} \mathrm{O}$. We further conclude that this nitrogen is present in the gas within and around the powder sample. This is the only hypothesis that can quantitatively explain the full spectrum of experimental observations:

- The linear scaling of 871-keV flux with nitric acid molarity.

- The similar 871-keV gamma-ray production rates for americium and plutonium alpha particles.

- The lack of an observable 871-keV flux for americium in a nitrogen-free solution.

- The lack of an observable 871-keV flux for a variety of plutonium oxide samples. (Martin, 1965; Marlow, 1999)

- The lack of an observable 871-keV flux for purified plutonium oxide powder in a nitrogen-free atmosphere.

- The reasonable scaling of the $871-\mathrm{keV}$ flux with nitrogen concentration and alpha-particle range for both aqueous and powder samples.

The "oxide" attribute should be re-evaluated in light of these results. While this attribute clearly retains some value, it has less than originally claimed. The implications of these results for arms control are complex. Clearly, the presence of an 871-keV gamma-ray flux indicates that a sample is not metallic. Unfortunately, the converse assertion is far from true. That is, the lack of a measurable $871-\mathrm{keV}$ gamma-ray flux does not necessarily indicate that the sample is metallic. Plutonium in the oxide form can easily be "disguised" either via compaction, purification, or atmospheric control. We would suggest that a more appropriate name for this attribute would be "presence of nitrogen." This new name accurately conveys the strengths and weaknesses of this attribute, as we now understand them.

\section{References}

J. M. Cleveland, "Plutonium Conversion Processes," Chapter 15 of Plutonium Handbook: A Guide to the Technology, Vol. 2, O.J. Wick, Ed., Gordon and Breach Science Publishers, New York, 1967.

Bruce D. Geelhood and Anthony J. Peurrung, Plutonium Oxide Measurement concerns for the Mayak/PPIA Demonstration, 25 August 1999.

Glenn F. Knoll, Radiation Detection and Measurement, $2^{\text {nd }}$ Ed., Wiley, New York, 1989. H.-H Hsu, Nuclear Instruments and Methods 193 (1982) p. 383.

Keith Marlow, Dean Mitchell and Thomas Laub, "Gamma-Ray Spectral Measurements of Plutonium Oxide", Sandia National Laboratories, 27 September, 1999. 
H.R. Martin, "Reaction Gamma Rays in Plutonium Compounds, Mixtures, and Alloys," Dow Chemical, Rocky Flats, RFP-2382, June 1965.

Anthony Peurrung, Richard Arthur, Bruce Geelhood, Sharon Pratt, Walter Hensley, Elwood Lepel, "Origin of the 871-keV line in Plutonium Oxide", Pacific Northwest National Laboratory, 30 September, 1999.

H. Zhou, X, Wang, C.. Wang, M. Hua, G. Huang, G. Fan, T. Lu, and S. Bartel, "Discrete Gamma Radiation in Interaction of $14.9 \mathrm{MeV}$ Neutrons with Lead," Nucl. Sci. \& Eng. 134 (2000) p. 106. 


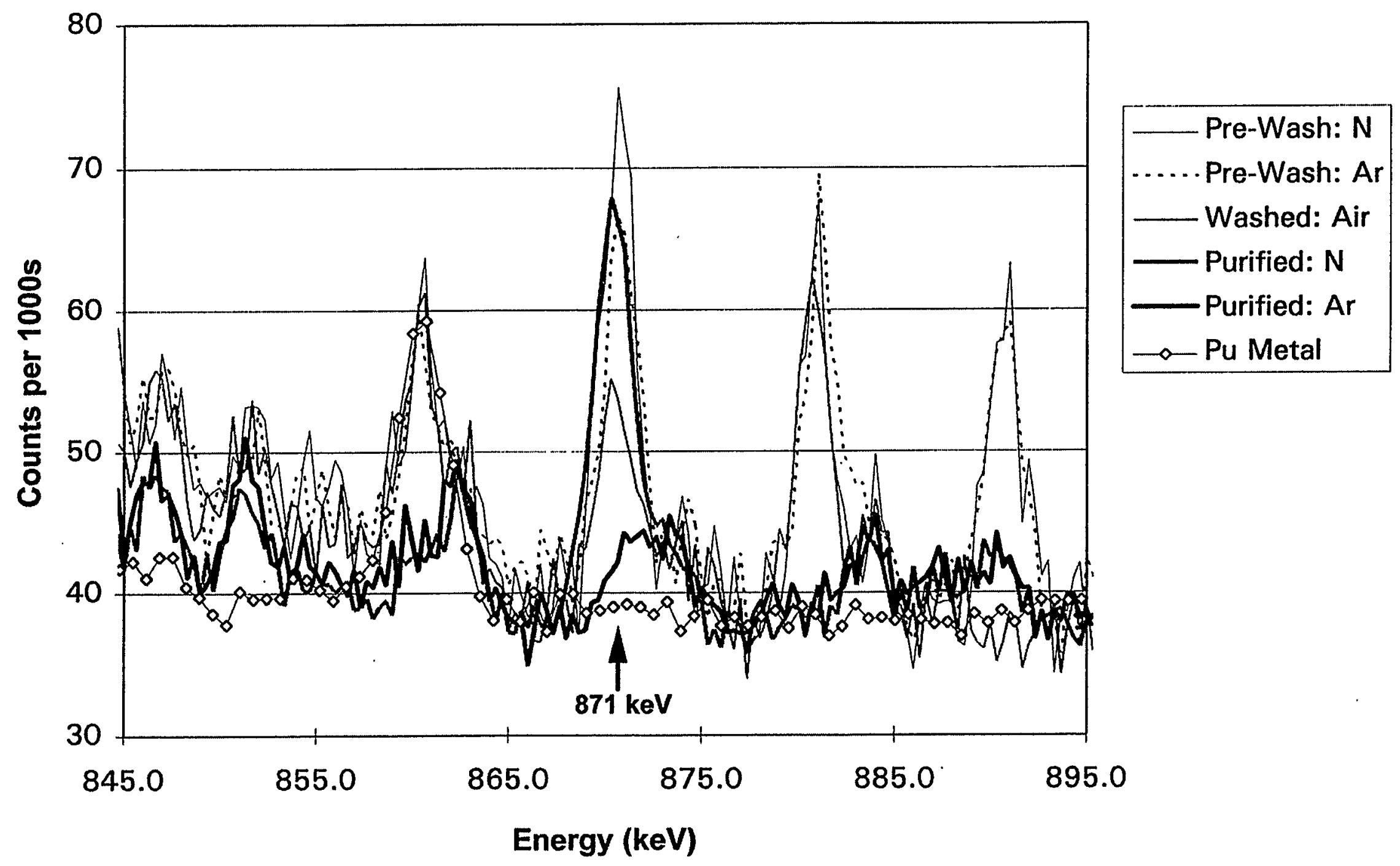

Figure 1. Comparison of Normalized Spectra for the Same Raw, Washed, and Purified Plutonium Oxide Sample. Please note that spectra for the purified sample are elevated by 10 counts/1000s for comparison. 


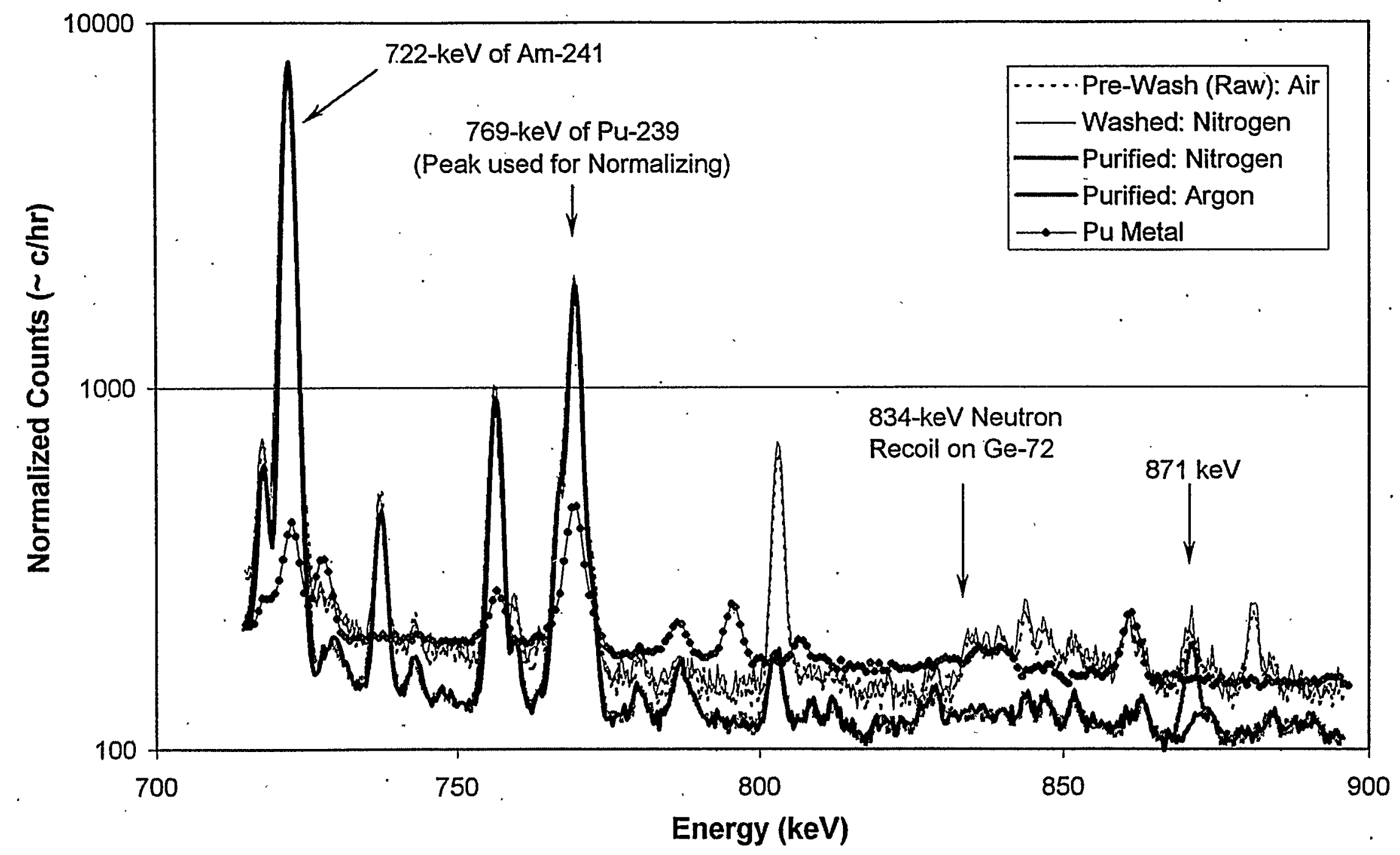

Figure 2. Comparison of Normalized Spectra for Raw, Washed, and Purified Plutonium Oxide Sample, Expanded to a Wider Energy Range. Note the arrow indicating the $769-\mathrm{keV}{ }^{239} \mathrm{Pu}$ peak used for normalization. 


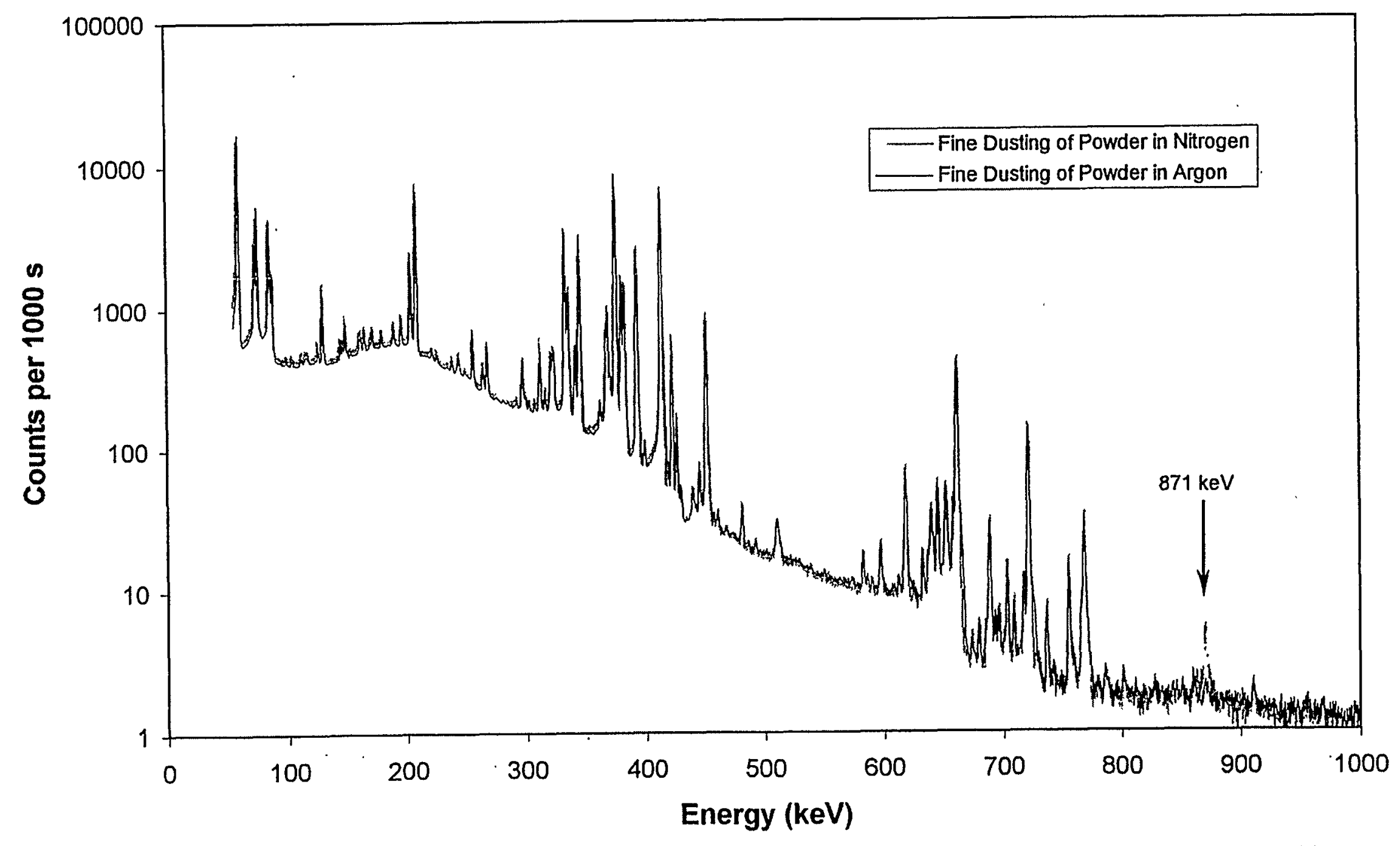

Figure 3 Normalized Spectra for Dusting of Purified Plutonium Oxide Powder under Argon and Nitrogen. Note the intensity of the $871-\mathrm{keV}$ line as the only major difference between the spectra. 


\section{DISTRIBUTION}

No. of

Copies

OFFSITE .

DOE

6

Andrew Bieniawski, NN-42

Michelle Smith, NN-42

Mike Newman, NN-42

David Spears, NN-20

Richard Comerford, SO-222

John Murphy, NN-44

DOD

1 TR Koncher

$\underline{\text { NSA }}$

1 John Rus Askren

$\underline{B N L}$

2

Leon Forman

Peter Vanier

DTRA

3

Carolyn Pura

Thomas Rutherford

David Squire

SAIC

2

John McNeilly, CVR

Ronald Weitz

LANL

4

Bill Johnson

Mark Mullens

Robert Scarlet

Nancy Jo Nicholas
LLNL

5

John Luke

Tom Gosnell

Zach Koenig

Ron Ott

Jim Morgan

$\underline{\text { SNL }}$

2

Dean Mitchell

Jerry Quinlan

ORNL

3

James Mullens

Alex Riedy

Lloyd Porter, Y12

Pantex

2

Wayne Kiehl

Leigh Bratcher

INEEL

1 Gus Cafree

ONSITE

PNNL

18

Jim Fuller

Bruce Geelhood

Richard Arthur (5)

Anthony Peurrung

Jennifer Tanner

Hal Unden

Rod Martin

Information Release_(7) 